\title{
Comparison of tyrosinase antibody, tyrosinase-related protein- 1 and -2 antibodies, melanin-concentrating hormone receptor antibody levels with autologous serum skin test and autologous plasma skin test results in patients with vitiligo
}

\author{
Abdullah Unal', Hatice Uce Ozkol ${ }^{2}$, Yasemin Bayram ${ }^{3}$ Necmettin Akdeniz ${ }^{4}$ \\ ${ }^{1}$ Abdullah Unal Private Dermatology Clinics, Istanbul, Turkey \\ 2Department of Dermatology, Faculty of Medicine, Yuzuncu Yil University, Van, Turkey \\ ${ }^{3}$ Department of Microbiology, Faculty of Medicine, Yuzuncu Yil University, Van, Turkey \\ ${ }^{4}$ Department of Dermatology, Faculty of Medicine, Medeniyet University, Istanbul, Turkey
}

Adv Dermatol Allergol 2021; XXXVIII (3): 473-479

DOI: https://doi.org/10.5114/ada.2020.93272

\begin{abstract}
Introduction: Although the exact etiopathogenesis of vitiligo is unknown, the autoimmunity hypothesis is much in evidence. The autologous serum skin test (ASST) and autologous plasma skin test (APST) are in vivo methods used in the diagnosis of some autoimmune diseases, which are easy and inexpensive to perform.

Aim: In this study, we investigated whether or not ASST and APST could determine autoimmunity in patients with vitiligo.

Material and methods: In this study, 30 vitiligo patients presenting to the dermatology outpatient clinic and 30 healthy volunteers without any known autoimmune diseases were included. Antibodies such as tyrosinase, tyrosinase-related protein-1 (TYRP1), tyrosinase-related protein-2 (TYRP2) and melanin-concentrating hormone receptor 1 (MCHR1) antibodies determined to be associated with vitiligo were examined. In addition, the association of these antibodies with the positivity of ASST and APST, which were suggested to be associated with autoimmunity, were examined.

Results: In our study, tyrosinase antibody was found to be significantly higher in vitiligo patients. ASST was positive in $12(40 \%)$ patients with vitiligo and $8(26.6 \%)$ control subjects. APST was positive in $8(26.6 \%)$ of the patients with vitiligo and in 2 (6.6\%) of the controls, and there was a significant difference between the groups in terms of APST positivity $(p=0.032)$. In addition, in our study, a significant correlation was found between TYRP1 antibody positivity and APST positivity in the patient group $(p=0.005)$.

Conclusions: These findings suggest that we may use APST to investigate the autoimmune etiopathogenesis of vitiligo.
\end{abstract}

Key words: vitiligo, autoimmunity, antibodies, autologous serum skin test.

\section{Introduction}

Vitiligo is an acquired pigmentation disorder of unknown cause with loss of melanocytes in the epidermis. Vitiligo is the most common cause of depigmentation worldwide, with a prevalence of $1 \%[1,2]$. The most common clinical form of vitiligo is fully amelanotic macules and patches surrounded by normal skin. The colour of these patches is usually uniformly chalkwhite or milky white. Recurrent trauma areas such as the dorsal surfaces of the hands and feet are the most common sites of involvement [3]. The most accepted classification in vitiligo today is the classification proposed by the European Task Force. Accordingly, vitiligo is divided into two groups as generalized and localized. Generalized vitiligo is divided into the acrofacial vitiligo and vitiligo vulgaris

Address for correspondence: Assoc. Prof. Dr. Hatice Uce Ozkol MD, Department of Dermatology, Faculty of Medicine, Yuzuncu Yil University, 6510 Van, Turkey, phone: +90 43221576 07, fax: +90 43221675 19, e-mail: drhaticeuce@gmail.com, haticeuceozkol@yyu.edu.tr Received: 15.01 .2020 , accepted: 3.02 .2020$. 
subtypes, whereas local vitiligo is divided into the segmental and focal vitiligo subtypes [4]. Histopathologically, there is a complete loss of melanocytes. Usually there is no inflammatory infiltrate [5].

Although the exact etiopathogenesis of the disease is unknown, autoimmune, genetic, nervous system and self-destruction hypotheses have been proposed [6]. Genetic factors, structural functional disorders in melanocytes and defects in melanocyte growth factors are other causes that have been implicated in the aetiology. The autoimmune theory is the most accepted one [7]. Autoimmune hypothesis is caused by disruption of self-tolerance. Thyroid diseases such as Hashimoto's thyroiditis and Graves' disease, diabetes mellitus, Addison's disease and, more rarely, other autoimmune diseases may be associated with vitiligo. In the autoimmune hypothesis, autoantibodies against melanocyte antigens lead to destruction of melanocytes by humoral immune mechanisms or cellular immune mechanisms such as cytotoxic T cells [8]. Melanin biosynthesis occurs with the tyrosinase, tyrosinase-related protein-1 and -2 (TYRP1 and TYRP2) enzymes. The antibodies that react with these proteins, which are specific for melanocytes, are antibodies that attract the most attention. Tyrosinase and tyrosinase-related proteins-1 and -2, melaninconcentrating hormone receptor 1 (MCHR1) antibodies are specific autoantibodies that are significant in vitiligo [9]. Tyrosinase, TYRP-1 and TYRP-2 are proteins that are key to controlling the formation of pigmentation, as well as being specifically accepted by melanosomes [10].

Autoimmunity has been reported to be involved in the aetiology of both chronic urticaria and vitiligo. Autologous serum skin test (ASST) and autologous plasma skin test (APST) are intradermal tests used in chronic urticaria [11]. ASST is an easy to perform and economical in vivo test that shows the presence of antibodies against FceRl $\alpha$ or lgE [11]. The presence of melanocyte autoantibodies in patients with vitiligo has been shown in many studies [12-15].

In this study, we investigated the relationship of ASST and APST positivity with tyrosinase, TYRP-1 and -2 and MCHR1 antibodies in vitiligo patients.

\section{Aim}

The aim of our comparison with these antibodies was to investigate whether ASST and APST, which are more cost-effective and easier to perform, present autoimmunity in patients with vitiligo.

\section{Material and methods}

Thirty patients diagnosed with vitiligo presenting to our dermatology outpatient clinic were included in the study. Thirty healthy individuals free from vitiligo and other autoimmune diseases were included in the study as the control group. Physical examination of the patients was performed and the diagnosis of vitiligo was confirmed with Wood's lamp examination. The patients' ages, occupations, diagnosis of the presenting disease, the systemic drugs they used and additional systemic diseases were recorded. People with no informed consent, younger than 7, older than 65 years old, people who had an autoimmune disease other than vitiligo or who used systemic corticosteroids or other immunosuppressive drugs, people who were diagnosed with urticaria and angioedema and people who used antihistamines for any reason were excluded from the study. Blood samples were drawn from patient and healthy control groups and placed in a gel tube with a yellow cap to look for the tyrosinase antibody, TYRP 1-2 antibodies and MCHR1 antibody. The blood samples were centrifuged at 5000 rpm for 20 min. The obtained serum was stored until the determination of the tyrosinase antibody, TYRP 1-2 antibodies and MCHR1 antibody at $-80^{\circ} \mathrm{C}$. Tyrosinase antibody, TYRP 1-2 antibodies and MCHR1 antibody levels were measured using ready-to-use kits with Microelisa. The ethics committee approval dated 27.02.2014 and numbered 03 was obtained from the Committee for Evaluation of Scientific Research for the study. Written informed consent forms were obtained from all patients and controls who participated in the study.

\section{Performing ASST and APST tests}

Autologous plasma skin and autologous serum skin tests were applied to the patient and healthy control groups. The flexor surface of both forearms were cleaned with swab before application. Prior to administration, the patients were asked to discontinue their long-acting antihistamines and mast cell stabilizers one week in advance and short-acting antihistamines 3 days in advance, if possible. Before the test, it was observed whether the patients had received any immunosuppressive drugs in the last 3 months or not. Adrenaline, antihistamines and prednisolone were made available before the test due to the risk of anaphylaxis and other allergic reactions. $5 \mathrm{ml}$ of venous blood were drawn from the patients and controls under sterile conditions to be placed in a sterile biochemistry and sodium citrate coagulation tube. The blood samples were allowed to stand at room temperature for $30 \mathrm{~min}$. Then the blood samples were centrifuged at $5000 \mathrm{rpm}$ for $20 \mathrm{~min}$, and the serum and plasma were separated. Histamine and saline of $0.1 \mathrm{ml}$ each were added to autologous plasma and undiluted autologous serum within an insulin syringe and applied intradermally to a hairless area on the volar face of the forearm being at least $3 \mathrm{~cm}$ between the injection sites. The test results were evaluated 30 min after the injection. An erythematous papule response with autologous serum and autologous plasma was evaluated as positive if its diameter were $1.5 \mathrm{~mm}$ or higher than the response to saline. After the statistical analysis of the obtained re- 
Table 1. Demographic characteristics of the participants in the study

\begin{tabular}{|c|c|c|c|c|}
\hline Vitiligo types & Gender & Average age & Family history & $\begin{array}{c}\text { Additional disease } \\
\text { history }\end{array}$ \\
\hline Focal: 2 (6.6\%) & \multirow{2}{*}{$\begin{array}{c}\text { All of the participants } \\
29(48.3 \%) \text { male } \\
31(51.7 \%) \text { female }\end{array}$} & \multirow[t]{2}{*}{ Controls: 30.30} & \multirow{2}{*}{$\begin{array}{c}9 \text { patients have familial } \\
\text { history }(30 \%)\end{array}$} & \multirow{2}{*}{$\begin{array}{c}4 \text { patients have } \\
\text { additional disease } \\
\text { history }\end{array}$} \\
\hline Generalized: 26 (86.6\%) & & & & \\
\hline Segmental: 1 (3.3\%) & \multirow{2}{*}{$\begin{array}{c}\text { Patients with vitiligo } \\
15(50 \%) \text { male } \\
15(50 \%) \text { female }\end{array}$} & \multirow[t]{2}{*}{ Vitiligo patients: 29.87} & \multirow{2}{*}{$\begin{array}{l}21 \text { patients have no } \\
\text { history (70\%) }\end{array}$} & \multirow{2}{*}{$\begin{array}{c}26 \text { patient have not } \\
\text { additional disease } \\
\text { history }\end{array}$} \\
\hline Variant type: 1 (3.3\%) & & & & \\
\hline
\end{tabular}

Table 2. The relationship between ASST and APST test results and antibody levels

\begin{tabular}{|c|c|c|c|c|c|c|c|c|}
\hline Variable & Tyrosinase & $P$-value & TYRP 1 & $P$-value & TYRP 2 & $P$-value & MCHR 1 & $P$-value \\
\hline Patients with positive ASST & 79.03 & NS & 17.57 & NS & 14.31 & NS & 11.71 & NS \\
\hline Patients with negative ASST & 69.29 & & 18.33 & & 14.25 & & 10.57 & \\
\hline Patients with positive APST & 81.84 & $\leq 0.05$ & 20.63 & $\leq 0.05$ & 15.46 & NS & 12.02 & NS \\
\hline Patients with negative APST & 60.81 & & 16.91 & & 14.07 & & 10.34 & \\
\hline Controls with positive ASST & 60.36 & NS & 16.83 & NS & 14.49 & NS & 12.02 & NS \\
\hline Controls with negative ASST & 61.26 & & 19.09 & & 15.78 & & 10.34 & \\
\hline Controls with positive APST & 62.79 & NS & 16.96 & NS & 14.59 & NS & 14.49 & NS \\
\hline Controls with negative APST & 60.81 & & 18.45 & & 15.63 & & 11.01 & \\
\hline
\end{tabular}

ASST - autologous serum skin test, APST - autologous plasma skin test, NS - nonspecific.

sults, an evaluation was performed whether there was a significant relationship between the parameters.

\section{Statistical analysis}

Among the features emphasized, descriptive statistics for continuous variables were expressed as mean, standard deviation, minimum and maximum values, while the categorical variables were expressed as numbers and percentages. The Mann-Whitney $U$ test was used to compare the group means for continuous variables. The $\chi^{2}$ test was used to determine the relationship between the groups and the categorical variables. The statistical significance level was accepted as 5\% in the calculations and the SPSS statistical package program was used for the calculations.

\section{Results}

Of the healthy control and the patient groups, $29(48.3 \%)$ subjects were male and 31 (51.7\%) were female. Of the patients with vitiligo, 15 (50\%) were male and 15 (50\%) were female. The mean age of the patient group was 29.87, while the mean age of the control group was 30.30. There was no statistically significant difference between the patients and the controls in terms of age. The youngest participant was 8 years old and the oldest participant was 62 years of age. Two (6.6\%) of the patients with vitiligo had focal, $26(86.6 \%)$ had generalized, 1 (3.3\%) had segmental and 1 (3.3\%) had variant vitiligo. There were no one with universal vitiligo in our patient group. Nine (30\%) of the vitiligo patients had a family history (Table 1). Tyrosinase antibody was positive in 23 (76.6\%) patients with vitiligo and in 12 (40\%) controls, and a significant difference was found between the groups in terms of tyrosinase antibody positivity ( $p=$ 0.004). MCHR1, TYRP1 and TYRP2 antibodies were found to be similar in the patient and the control groups and no significant difference was observed (Table 2).

Forty $(66.6 \%)$ of all participants included in the study were ASST-negative and 20 (33.3\%) were positive. APST was positive in 10 (16.6\%) of the participants and negative in 50 (83.3\%). ASST was positive in 12 patients and in 8 controls, but there was no significant difference in ASST positivity between the groups. APST was positive in 8 patients and in 2 controls, and there was a significant difference between the groups in terms of APST positivity $(p=0.032)$ (Table 3). There was no significant difference

Table 3. Test results of all participants

\begin{tabular}{lccc}
\hline Variable & Patient & Control & P-value \\
\cline { 1 - 2 } Negative ASST & 18 & 22 & NS \\
\cline { 1 - 2 } Positive ASST & 12 & 8 & \\
\cline { 1 - 2 } Positive APST & 8 & 2 & $\leq 0.05$ \\
\cline { 1 - 2 } Negative APST & 22 & 28 & \\
\hline
\end{tabular}

ASST - autologous serum skin test, APST - autologous plasma skin test, NS - nonspecific. 
Table 4. ASST and APST results according to clinical types of vitiligo

\begin{tabular}{lccccc}
\hline Variable & & \multicolumn{2}{c}{ Vitiligo clinical types } \\
\cline { 3 - 6 } & & Focal & Generalized & Segmental & Variant \\
\hline Negative APST & 22 patients & 1 & 21 & 0 & 0 \\
\hline Positive APST & 8 patients & 1 & 5 & 1 & 1 \\
\hline Negative ASST & 18 patients & 1 & 16 & 0 & 1 \\
\hline Positive ASST & 12 patients & 1 & 10 & 1 & 0 \\
\hline
\end{tabular}

in the ASST and APST results according to the clinical types of vitiligo (Table 4).

No significant relationship was found between tyrosinase antibody positivity and negativity, and ASST positivity and negativity in the patient group and the control group. No significant correlation was found between MCHR1 antibody positivity and negativity and ASST and APST positivity and negativity in vitiligo patients. However, there was a statistically significant relationship between TYRP1 and tyrosinase antibody positivity and negativity and APST positivity and negativity in the patient group ( $p=0.005$ ) (Table 2). However, this relationship was not detected for ASST. No significant correlation was found between TYRP2 antibody positivity and negativity and ASST and APST positivity and negativity in the patient group. The ASST and APST results of patients according to gender and mean age have been demonstrated in Table 5.

\section{Discussion}

Although known for a long time, the aetiology of vitiligo has not been clarified yet. Genetic, autoimmune, nervous system and self-destruction hypotheses have been proposed in the formation of the disease. Langerhans cells, free radicals, melanocyte growth factors, melatonin receptor, viral diseases and apoptosis are other relevant factors [6]. Both humoral and cellular immunity changes occur in patients with vitiligo [13]. Zamani et al. suggested that vitiligo was associated with autoimmune thyroid disease, pernicious anaemia, Addison's disease

Table 5. ASST and APST results according to gender and age of patients

\begin{tabular}{lccc}
\hline Variable & Average age & Male & Female \\
\hline $\begin{array}{l}\text { Patients with } \\
\text { positive ASST }\end{array}$ & 35.42 & 11 & 9 \\
\hline $\begin{array}{l}\text { Patients with } \\
\text { negative ASST }\end{array}$ & 26.17 & 18 & 22 \\
\hline $\begin{array}{l}\text { Patients with } \\
\text { positive APST }\end{array}$ & 30.75 & 6 & 4 \\
\hline $\begin{array}{l}\text { Patients with } \\
\text { negative APST }\end{array}$ & 29.55 & 23 & 27 \\
\hline
\end{tabular}

and systemic lupus erythematosus. In the same study, it was reported that these diseases were observed at an increased frequency in first-degree relatives of vitiligo patients [16].

The most powerful association of organ-specific autoantibodies in vitiligo patients was seen with autoimmune thyroiditis with a frequency of 30\% [17]. While generalized or focal non-dermatomal vitiligo is often associated with the autoimmune hypothesis, segmental vitiligo is mostly associated with the neural hypothesis [9]. However, in our study, patients with autoimmune diseases were excluded from the study. The reason for us not having included patients with additional autoimmune diseases was to prove the exact relationship between the presence of antibodies and skin tests. We think that this is the reason for our results being different from the previous studies.

Antibodies to melanocytes are present in the circulation of the majority of patients with vitiligo $[18,19]$. Although a broad spectrum of antigens has been reported in the targets of vitiligo antibodies, none have yet been non-arguably identified as the major autoantigen of the disease. In conclusion, the contribution of anti-melanocyte antibodies in the pathogenesis of vitiligo is not fully understood and it is still possible that they have no role in the aetiology of vitiligo [12].

Tyrosinase is the most important enzyme that plays a key role in melanin synthesis [20]. In a study with 21 patients with vitiligo and 20 healthy controls, $42.8 \%$ of the patients had antibodies to tyrosinase. No antibody response was observed in the control group. No significant correlation was found between the presence of antibodies and age and gender [21]. Again, tyrosinase antibody was found to have different positivity rates of $61 \%$ and $39 \%$ in different studies in the literature $[12,14]$. However, tyrosinase antibodies could not be detected in Xie et al.'s study [22]. In one study, tyrosinase antibody was found to be positive in 11 of 79 vitiligo patients [23]. In another study, the rate of tyrosinase antibody was reported as $11 \%$ in patients with generalized vitiligo. No significant relationship was reported between antibody positivity and gender [24]. In our study, we detected tyrosinase antibody positivity in 23 (76.6\%) patients with vitiligo and in 12 (40\%) controls and found higher positiv- 
ity rates compared to previous studies in the literature. In our study, we found that tyrosinase antibody positivity was significantly higher compared to the control group. Considering that tyrosinase is the most important enzyme in melanin production, the results can be considered significant.

In a study that did not exclude patients with vitiligo and with accompanying autoimmune diseases, 24 out of 145 patients diagnosed with vitiligo had MCHR1 positive results. Significant differences were found between the vitiligo patient group and the control group in terms of MCHR1 antibody positivity. In addition, there was no correlation between MCHR1 antibody positivity-negativity and gender [25]. In another study, MCHR1 antibody was found to be positive in 14 (18\%) of 79 vitiligo patients [23]. In another study with vitiligo patients and healthy controls, MCHR1 antibody was shown in $42.8 \%$ of patients. No antibody response was seen in the control group. No significant correlation was found between the presence of antibodies and age and gender [21]. In one study, 9/55 (16.4\%) of vitiligo patients had antibodies to MCHR1 [26]. TYRP1 is another enzyme involved in melanin synthesis [27]. In one study, 5.7\% of vitiligo patients had TYRP1 positivity. In contrast, TYRP1 was negative in the whole control group [15]. In a study by Michelsen, TYRP1 antibody positivity was reported as $5 \%$ in vitiligo patients [28]. Autoantibodies against TYRP1 and melanosomal matrix protein gp100 (Pmel17) have been identified in only a small proportion, as $5 \%$ of individuals with vitiligo $[15,29]$. In one study, TYRP1 antibody was reported as $4 \%$ in vitiligo patients. There was no significant relationship between antibody positivity and gender [24]. Differences in detection rates of antibodies to these melanocyte proteins may be related to the group of patients with vitiligo studied [12]. Fifty-three vitiligo patients and 20 healthy controls were included in a study in which TYRP2 antibody was examined. TYRP2 antibody was reported to be positive in $5 \%$ of 53 vitiligo patients and negative in all controls [20]. One study found anti-TYRP2 antibodies as $67 \%$ in vitiligo patients [12]. In another study, TYRP2 antibody was reported as $4 \%$ in vitiligo patients. There was no significant relationship between antibody positivity and gender [24]. In our study, only tyrosinase antibody was found to be significantly higher. However, other antibody values were not significantly positive. Since vitiligo patients with autoimmune disease were excluded from our study, the antibody levels may be similar in the patient and the control groups. Our aim to exclude autoimmune diseases was to prove whether or not the results of in vivo ASST and APST showed direct vitiligo antibodies.

IgG-type antibodies leading to histamine release have been shown to be autoantibodies against FC\&RI in the majority of patients with chronic urticaria (CU). In a small number of cases, there are autoantibodies against the IgE molecule itself [30]. Anti-FC\&RI autoantibodies were detected in diseases such as pemphigus vulgaris (39\%), dermatomyositis (36\%), systemic lupus erythematosus (20\%) and bullous pemphigoid (13\%) except urticaria [30]. ELISA and immunoblot methods have shown that these antibodies can be found in normal individuals as well [31]. The autologous serum test is a sensitive and specific intradermal test. Positive ASST demonstrates the autoimmune pathogenesis of urticaria in clinical practice [32]. The use of plasma instead of autologous serum has been shown to result in a higher rate of positive response in the intradermal skin test [33]. In APST, it is recommended to use sodium citrate as an anticoagulant.

There are studies reporting that ASST-positive CU patients are associated with autoimmune diseases such as autoimmune thyroiditis, type 1 diabetes mellitus, rheumatoid arthritis, Graves' disease and celiac disease, and that autoimmune markers such as anti-thyroid, rheumatoid factor and antinuclear antibodies are increased in ASST-positive CU patients [34]. In addition, FCR was shown to play an important role in the pathogenesis of these diseases in experimental models of autoimmune diseases [35]. In the animal model study by Trcka et al., it was observed that the autoimmune pathogenesis of vitiligo progressed faster in Fc- and complement-deficient mice [36]. In a study by Fiebiger et al., anti-FCERI autoantibodies were detected in 38\% of 281 patients with CU, in $36 \%$ of patients with dermatomyositis, in $39 \%$ of patients with pemphigus vulgaris, in $20 \%$ of patients with systemic lupus erythematosus (SLE), and in 13\% of patients with bullous pemphigoid [37].

Many authors acknowledge that autoantibodies are an important etiopathogenetic factor in vitiligo. However, it is very expensive to test for antibodies in routine laboratory conditions. In our study, we investigated whether in vivo tests, ASST and APST, were related to antibody levels without using these expensive tests. In our study, while ASST was positive in $12(40 \%)$ patients with vitiligo and in 8 (26.6\%) controls, and ASST positivity was significantly higher in vitiligo patients, there was no statistically significant difference between the groups in terms of ASST positivity. However, this result may have been obtained due to the fact that patients with additional autoimmune disease had been excluded from the study.

In our study, APST was positive in 8 (26.6\%) patients and in 2 (6.6 controls \%), and a statistically significant difference was found between the groups in terms of APST positivity $(p=0.032)$. This finding is consistent with the results of studies in the literature suggesting the superiority of APST over ASST in determining autoimmunity in the etiopathogenesis of CU.

In our literature review, we did not find any information regarding either ASST or APST application in vitiligo patients. Therefore, we could not compare our data since there were no similar studies.

Furthermore, in our study, a significant correlation was found between TYRP1 antibody positivity and negativity and APST positivity and negativity in the patient 
group. APST was found to be significantly highly positive in patients with TYRP1 antibody positivity. This finding suggests that APST can be used to investigate autoimmunity in vitiligo patients.

\section{Conclusions}

In our study, we observed that out of tyrosinase, TYRP1, TYRP2 and MCHR1 antibodies, only the tyrosinase antibody was highly positive in vitiligo patients. A statistically significant difference was found between vitiligo patients and healthy controls in terms of APST positivity. In addition, a significant correlation was found between TYRP1 antibody positivity and negativity and APST positivity and negativity in the patient group. This finding suggests that we can use APST to investigate the autoimmune etiopathogenesis of vitiligo.

\section{Acknowledgments}

Van YYU - BAP provided financial support to this clinical trial.

\section{Conflict of interest}

The authors declare no conflict of interest.

\section{References}

1. Ezzedine K, Eleftheriadou V, Whitton M, et al. Vitiligo. Lancet 2015; 386: 74-84.

2. Tarlé RG, Silva de Castro CC, et al. Polymorphism of the Ecadherin gene $\mathrm{CDH} 1$ is associated with susceptibility to vitiligo. Exp Dermatol 2015; 24: 300-2.

3. Ortonne JP. Vitiligo and other disorder hypopigmentation. In: Dermatology. Callen JP, Horn TD, Mancini AJ, et al. (eds.). Mosby Elsewier, USA 2008; 913-20.

4. Taïeb A, Picardo M; VETF Members. The definition and assessment of vitiligo: a consensus report of the Vitiligo European Task Force. Pigment Cell Res 2007; 20: 27-35.

5. Habif TP. Vitiligo. In: Clinical Dermatology. Fifth edition. Habif TP (ed.). Mosby Elsewier, USA 2010; 765-9.

6. Borlu M. Etiopathogenesis of vitiligo. Turkiye Klinikleri J Dermatol Special Topics 2009; 2: 8-13.

7. Gauthier Y, Cario Andre M, Taïeb A. A critical appraisal of vitiligo etiologic theories. Is melanocyte loss a melanocytorrhagy? Pigment Cell Res 2003; 16: 322-32.

8. Glassman SJ. Vitiligo, reactive oxygen species and T-cells. Clin Sci (Lond) 2011; 120: 99-120.

9. Ongenae K, Van Geel N, Naeyaert JM. Evidence for an autoimmune pathogenesis of vitiligo. Pigment Cell Res 2003; 16: 90-100.

10. Schallreuter KU. Vitiligo. In: Autoimmune Diseases of the Skin. Hertl M (ed.). Springer Link 2005; 367-81.

11. Yeşilova Y, Kaçar SD, Sula B, Uçmak D. Autologous serum and plasma test results of the chronic urticaria patients in the South Eastern Anatolia Turkey. Asthma Allergy Immunol 2011; 9: 73-8.

12. Kemp EH, Gavalas NG, Gawkrodger DJ, Weetman AP. Autoantibody responses to melanocytes in the depigmenting skin disease vitiligo. Autoimmun Rev 2007; 6: 138-42.
13. Rocha IM, Oliveira LJ, De Castro LC, et al. Recognition of melanoma cell antigens with antibodies present in sera from patients with vitiligo. Int I Dermatol 2000; 39: 840-3.

14. Baharav E, Merimski O, Shoenfeld Y, et al. Tyrosinase as an autoantigen in patients with vitiligo. Clin Exp Immunol 1996; 105: 84-8.

15. Kemp EH, Waterman EA, Gawkrodger DJ, et al. Autoantibodies to tyrosinase-related protein-1 detected in the sera of vitiligo patients using a quantitative radiobinding assay. Br J Dermatol 1998; 139: 798-805.

16. Zamani M, Spaepen M, Sghar SS, et al. Linkage and association of HLA class II genes with vitiligo in a Dutch population. Br J Dermatol 2001; 145: 90-4.

17. Huggins RH, Schwartz RA, Janniger CK. Vitiligo. Acta Dermatovenerol Alp Panonica Adriat 2005; 14: 137-42.

18. Farrokhi S, Hojjat-Farsangi M, Noohpisheh MK, et al. Assessment of the immune system in 55 Iranian patients with vitiligo. J Eur Acad Dermatol Venereol 2005; 19: 706-11.

19. Naughton GK, Eisinger M, Bystryn JC. Antibodies to normal human melanocytes in vitiligo. J Exp Med 1983; 158: 246-51.

20. Kemp EH, Gawkrodger DJ, Watson PF, et al. Immunoprecipitation of melanogenic enzyme autoantigens with vitiligo sera: evidence for cross-reactive autoantibodies to tyrosinase and tyrosinase-related protein-2 (TRP-2). Clin Exp Immunol 1997; 109: 495-500.

21. Kroon MW, Kemp EH, Wind BS, et al. Melanocyte antigenspecific antibodies cannot be used as markers for recent disease activity in patients with vitiligo. J Eur Acad Dermatol Venereol 2013; 27: 1172-5.

22. Xie Z, Chen D, Jiao D, et al. Vitiligo antibodies are not directed to tyrosinase. Arch Dermatol 1999; 135: 417-22.

23. Kemp EH, Emhemad S, Akhtar S, et al. Autoantibodies against tyrosine hydroxylase in patients with non-segmental (generalised) vitiligo. Exp Dermatol 2011; 20: 35-40.

24. Pradhan V, Patwardhan M, Thakkar V, et al. Vitiligo patients from India (Mumbai) show differences in clinical, demographic and autoantibody profiles compared to patients in western countries. J Eur Acad Dermatol Venereol 2013; 27: 279-86.

25. Zhou M, Guan C, Lin F, et al. Immunodetection of the MCHR1 antibody in vitiligo patient sera. Int J Mol Med 2011; 27: 725-9.

26. Gottumukkala RV, Waterman EA, Herd LM, et al. Autoantibodies in vitiligo patients recognize multiple domains of the melanin-concentrating hormone receptor. J Invest Dermatol 2003; 121: 765-70.

27. Cui TT, Yi XL, Zhang WG, et al. miR-196a-2 rs11614913 polymorphism is associated with vitiligo by affecting heterodimeric molecular complexes of Tyr and Tyrp1. Arch Dermatol Res 2015; 307: 683-92.

28. Michelsen D. The Double Strike Hypothesis of the vitiligo pathomechanism: new approaches to vitiligo and melanoma. Med Hypotheses 2010; 74: 67-70.

29. Kemp EH, Gawkrodger DJ, Watson PF, et al. Auto-antibodies to human melanocyte-specific protein Pmel17 in the sera of vitiligo patients: a sensitive and quantitative radioimmunoassay (RIA). Clin Exp Immunol 1998; 114: 333-8.

30. Utas S. Autoimmune urticaria. Türkiye Klinikleri J DermatolSpecial Topics 2008; 1: 12-9.

31. Sabroe RA, Greaves MW. Chronic idiopathic urticaria with functional autoantibodies: 12 years on. Br I Dermatol 2006; 154: 813-9.

32. Engin B, Özdemir M. Evaluation of autologous serum skin test in chronic urticaria: a controlled retrospective study. Turkiye Klinikleri J Dermatol 2008; 18: 1-4. 
33. Aydın F, Yüksel EP. Diagnosis and treatment of autoimmune urticaria. Turkiye Klinikleri J Dermatol Special Topics 2012; 5: 16-9.

34. Konstantinou GN, Asero R, Maurer M, et al. EAACI/GA(2)LEN task force consensus report: the autologous serum skin test in urticaria. Allergy 2009; 64: 1256-68.

35. Ravetch JV, Clynes RA. Divergent roles for Fc receptors and complement in vivo. Annu Rev Immunol 1998; 16: 421-32.

36. Trcka J, Moroi Y, Clynes RA, et al. Redundant and alternative roles for activating $\mathrm{Fc}$ receptors and complement in an antibody-dependent model of autoimmune vitiligo. Immunity 2002; 16: 861-8.

37. Fiebiger E, Hammerschmid F, Stingl G, et al. Anti-FcepsilonRlalpha autoantibodies in autoimmune-mediated disorders. Identification of a structure function relationship. J Clin Invest 1998; 101: 243-51. 